Running head: REPLICATION OF APOCALYPSE SOON

The Effects of Dire and Solvable Messages on Belief in Climate Change:

A Replication Study

\author{
Jan G. Voelkel ${ }^{\mathrm{a}}$ \\ Adina T. Abeles ${ }^{\mathrm{b}}$ \\ Matthew Feinberg ${ }^{\mathrm{c}}$ \\ Robb Willer ${ }^{\mathrm{a}}$
}

a: Department of Sociology, Stanford University, Stanford, USA

b: Department of Communication, Stanford University, Stanford, USA

c: Rotman School of Management, University of Toronto, Toronto, Canada

Draft Date: April 8, 2021

Email addresses: jvoelkel@stanford.edu, abeles@alumni.stanford.edu, matthew.feinberg@rotman.utoronto.ca,willer@stanford.edu Article type: Preregistered Direct Replication

\title{
Funding Statement
}

The authors received funding for this project from the Stanford Center on Philanthropy and Civil Society. 


\begin{abstract}
Skepticism about climate change persists in the general public. Psychologists disagree on whether dire messages (emphasizing negative consequences) and solvable messages (emphasizing potential solutions) interact to increase or reduce belief in climate change, and whether the effects depend on recipients' psychological dispositions. A highly cited paper in this literature (Feinberg \& Willer, 2011) found that dire and solvable messages decreased, whereas dire and unsolvable messages increased climate change skepticism among people with a strong but not among those with a weak - belief in a just world. However, the validity of these influential results needs to be reassessed due to the underpowered nature of the original studies and shifting public opinion about climate change. In the current study, we propose several highly powered (total $n=6860$ ) conceptual and direct replications of Feinberg and Willer (2011).
\end{abstract}

Keywords: climate change; just world; framing; political psychology; replication 


\section{Statement of Relevance}

Climate change presents a major, global challenge to individual and societal well-being, yet skepticism regarding climate change persists in the American public. This replication study assesses the effectiveness of dire messages combined with solvable messages to impact belief in climate change. The results will speak to debates about whether dire messages reduce belief in climate change, increase belief in climate change, whether the effects depend on recipients' belief in a just world, and whether solvable messages are more effective. Practically, advocates and journalists need to know the consequences of these two common, yet very different, messaging approaches to communicate effectively with the mass public. 


\section{The Effects of Dire and Solvable Messages on Belief in Climate Change:}

\section{A Replication Study}

There is overwhelming scientific evidence that human activities cause climate change, and that climate change has dangerous implications for the world (Intergovernmental Panel on Climate Change, 2014). Nonetheless, skepticism about climate change persists in the mass public (Ballew et al., 2019). While psychological research has identified reasons why the public is not as engaged as the science suggests is warranted (Wong-Parodi \& Feygina, 2020), the question of how to increase public belief in climate change remains an active area of research.

One influential explanation (Feinberg \& Willer, 2011), focusing on people who strongly believe in a just world, is that dire messages (i.e., messages emphasizing negative consequences) increase belief in climate change if they are combined with solvable messages (i.e., messages emphasizing potential solutions) but undermine belief in climate change if they are paired with unsolvable messages (i.e., messages emphasizing the lack of potential solutions for climate change). This finding has shaped the academic discourse on climate change messaging (e.g., Hornsey \& Fielding, 2017; Markowitz \& Shariff, 2012; Van Boven, Ehret, \& Sherman, 2018). Further, journalists commonly use both dire messages, ranging from calls for a state of climate emergencies (Al Jazeera, 2020) to an inevitable climate catastrophe (Wallace-Wells, 2017), and solvable messages emphasizing solutions such as science, innovation, and international action (The New York Times Editorial Board, 2019). The current research questions whether dire messages and solvable messages really affect public belief in climate change, suggesting that other strategies for increasing belief in climate change must be developed.

\section{Belief in a Just World, Dire and Solvable Messages, and Belief in Climate Change}


One influential paper on the effect of dire and solvable messages on belief in climate change is Feinberg and Willer (2011). Their model (Figure 1) suggests that the effects of dire and solvable messages depend on recipients' belief in a just world. The argument is that dire and unsolvable messages foster beliefs that climate change is a serious threat that is impossible to solve and, thus, contradict the belief that the world is just. In contrast, dire and solvable messages do not violate the belief in a just world. Consequently, the effects of these messages are predicted to differ based on recipients' belief in a just world. Consistent with this theory, Feinberg and Willer (2011) found that people with a strong belief in a just world decreased their belief in climate change when reading about the dire and irreversible consequences of climate change but increased their belief in climate change when a message about dire consequences offered potential solutions for climate change. In contrast, climate change beliefs among people with a weak belief in a just world were not affected by such messages.

This paper, which has been cited over 400 times, made several contributions to the literature on climate change beliefs. First, it introduced the climate change communication literature to the idea that the public needs psychological "carrots" instead of only "sticks" (Markowitz \& Shariff, 2012; Pearce et al., 2015), encouraging researchers to explore not only negative emotions such as fear but also positive emotions such as hope (Stern, 2012; Westoby \& McNamara, 2019). Second, it advanced the idea that the effects of climate change messaging depend on the recipients' psychological dispositions (Hornsey \& Fielding, 2017; Van Boven et al., 2018), particularly emphasizing the relevance of beliefs about the world being just and stable (McCright et al., 2016).

\section{Alternative Models Predicting (Non-)Effects of Dire and Solvable Messages}


However, there are multiple reasons to suspect that the Feinberg \& Willer (2011) findings may not replicate. One possibility is that dire and solvable messages affected people's belief in climate change a decade ago but are no longer impactful because most people already believe in climate change by now (Ballew et al., 2019), a ceiling effect. Another possibility why dire and solvable messages were effective a decade ago but are no longer impactful is that people have been accustomed to these kinds of messages, a habituation effect (Groves \& Thompson, 1970). As a result of repeated exposure to dire messages and solvable messages, these messages may no longer influence belief in climate change (based on Petty \& Cacioppo, 1984). Note that this habituation hypothesis suggests that effects of dire and solvable messages could appear for newer issues. Finally, it is well known that many psychological effects do not replicate (Open Science Collaboration, 2015). Because the original studies in Feinberg and Willer (2011) were underpowered, we must consider the possibility that the original effects were false positives.

If the original finding in Feinberg and Willer (2011) do not hold, dire messages and solvable messages may still impact people's belief in climate change in other ways. Our research also tests the extended parallel process model (EPPM, Witte, 1992; see Figure 2). This model was developed in the health communication domain, is supported by multiple meta-analyses in this domain (Peters, Ruiter, \& Kok, 2013; Witte \& Allen, 2000), and is widely cited in the climate change literature (e.g., Feldman \& Hart, 2016; O’Neill \& Nicholson-Cole, 2009). Applied to climate change, this model suggests that dire messages paired with unsolvable messages would reduce belief in climate change, but dire messages paired with solvable messages would increase belief in climate change. The EPPM differs from the Feinberg and Willer (2011) model in that the EPPM considers recipients' belief in a just world as a moderator. Furthermore, while the model by Feinberg and Willer proposes a cognitive mechanism, the 
mechanisms in the EPPM are both cognition and emotion based. The model suggests that communicating the dire consequences of climate change elicits a threat which causes fear in the recipient. If the message does not communicate ways to address the source of the fear, the recipient will reduce their fear by questioning the source of the fear. However, if the fear-inducing message does communicate how the source of the fear can be addressed, the recipient will perceive a sense of efficacy and might feel more hope, resulting in a higher willingness to believe in the source of the fear.

\section{The Present Research}

In the current research, we propose a package of six studies to test these diverging hypotheses about the effects of dire and solvable messages on belief in climate change. First, we will conduct preregistered direct replications of the two original studies in Feinberg and Willer (2011). Second, because Feinberg and Willer only tested the effects of a dire and unsolvable message and a dire and solvable message, we report the results of three conceptual replication studies that separately manipulated the dire and solvable message components to identify the causal impact of both components. Finally, we will conduct another preregistered conceptual replication in which we replace the issue of climate change with a less well-known issue to address the habituation effect explanation. This package of studies allows us to fully examine the interplay of the effects of belief in a just world, dire messages, and solvable messages.

\section{Study 1}

Study 1 is a direct replication of Feinberg and Willer's (2011) first study. The goal of Study 1 is to test our hypotheses with the original materials. Note that the original studies only used a dire and unsolvable message and a dire and solvable message so that the effect of dire (vs less dire) messages cannot be estimated (Studies 3 to 5 will address this concern). 


\section{Method}

\section{Ethics and Reproducibility}

All studies will be approved by the Institutional Review Board at Stanford University. All participants will provide informed consent. All anonymized data files (including descriptions of how the original files were anonymized), study materials, and analysis scripts will be available via the Open Science Framework at https://osf.io/rqukf/?view_only=ddb97c31b8334ea0875df62b17150654. The preregistrations for Studies 1, 2, and 6 will also be available via the Open Science Framework.

\section{Participants}

We will recruit 888 participants from Amazon Mechanical Turk. We conducted a priori power analyses for a within-between interaction effect using $\mathrm{G}^{*}$ Power (Faul, Erdfelder, Buchner, \& Lang, 2009). Because large-scale replication studies (Open Science Collaboration, 2015; Klein et al., 2018) suggest that effect sizes in replication studies are typically a quarter to half the magnitude of the original effect size, we set $f=0.06$ (a quarter of the effect size in the original study $f=0.22$ ). A final sample size of 888 participants will provide us with $95 \%$ power given $\alpha$ $=.05$, two repeated measurements, four groups ${ }^{1}$, and assuming a correlation of $r=.63$ between the repeated measurements as in the original study.

Instead of recruiting undergraduate students from the same institution as in the original study, we will recruit participants from Amazon Mechanical Turk. This deviation is necessary because none of us works any longer at the institution where the original study was conducted, and it would not be feasible to recruit enough undergraduate students to reach our target sample size. One might also see this deviation as an improvement because MTurk samples are attentive

\footnotetext{
${ }^{1}$ Because we plan on conducting robustness checks with a dichotomized measure of belief in a just world, we treated belief in a just world as a factor with two groups. Note that treating it as a continuous measure should further increase our power.
} 
(Hauser \& Schwarz, 2016), show similar psychological dispositions with regard to political variables as the general public (Clifford, Jewell, \& Waggoner, 2015), and provide experimental effects that generalize to representative samples (Coppock, 2019). We will further assure the attentiveness of the sample by using multiple quality control checks. Specifically, we will recruit participants from a large panel of previously recruited MTurk workers. Participants will be excluded participants if they have duplicate IDs (keeping only the first case), fail at least one out of our two attention checks completed pre-treatment-assignment, leave the study before they are randomly assigned to a condition, or have missing values for our measures of belief in a just world or belief in climate change. To ensure sufficient variance in belief in climate change, the sample will be recruited with soft quotas for participants' political ideology: $40 \%$ liberal, $20 \%$ moderate, and $40 \%$ conservative.

\section{Materials and Procedure}

The materials and procedure are taken from Study 1 of Feinberg and Willer (2011). Any deviations from the original study are explicitly mentioned. The study will include two parts. At Time 1, participants will complete a questionnaire containing demographics, attention checks, and a seven-item measure of belief in climate change. Although the original study used a two-item measure, we will use the seven-item measure the original paper used in Study 2 to reduce measurement error.

At Time 2, three to four weeks later, participants will first complete a six-item General Belief in a Just World scale (Dalbert, Montada, \& Schmitt, 1987). Although the original study measured participants' belief in a just world at Time 1, we moved it to the beginning of Study 2 to avoid change over time and minimize measurement error. Next, participants will be randomly assigned to one of two article conditions. The manipulation is identical to the one used by 
Feinberg and Willer (2011). The first four paragraphs of these two articles will be identical and provide information about the dire consequences of climate change. In the dire and unsolvable message condition, the final two paragraphs argue that the consequences of climate change are irreversible. In the dire and solvable message condition, the final two paragraphs argue that there are still potential solutions to climate change. After the manipulation, participants will complete the same measure of belief in climate change as during the Time 1 survey. In addition, we will collect new, exploratory three-item measures of participants' perceptions of the argued threat of climate change and argued efficacy to address climate change as well as participants' experiences of fear and hope. More details on the manipulation and measures are available in the Supplementary Materials.

\section{Results}

In this and all following studies, we will use multiple linear regression analyses as our default analyses. The measure of belief in a just world will be standardized and always included in all regression models. Belief in climate change will be recoded to range from zero to one. We will control for gender, age, race, and education to maximize power. In Study 1, we will use the difference score (Time 2 - Time 1) in belief in climate change as the dependent variable. This will allow us to replicate the original findings while conducting additional analyses within the same analytical framework.

\section{Test of the Feinberg and Willer Model}

We will test the belief in a just world $\mathrm{x}$ message condition interaction effect predicted by the Feinberg and Willer model. This model further predicts the following pattern of simple effects. First, participants with a weak belief in a just world are not affected by the message condition and do not change their belief in climate change. Second, participants with a strong 
belief in a just world in the dire and unsolvable message condition will decrease their belief in climate change. Third, participants with a strong belief in a just world in the dire and solvable message condition will increase their belief in climate change.

\section{Test of the Extended Parallel Process Model}

We will test the main effect of message condition predicted by the EPPM. This model further predicts the following effects. First, participants in the dire and unsolvable message condition will decrease their belief in climate change. Second, participants in the dire and solvable message condition will increase their belief in climate change.

\section{Ceiling Effect}

We will test if the level of belief in climate change was higher than in the original studies and if there was sufficient room for increases in belief in climate change. In Feinberg and Willer's (2011) first study, the average belief in climate change was $M=0.73$ before the treatment and $M=0.74$ after the treatment. In Feinberg and Willer's (2011) second study, the average belief in climate change was $M=0.74$ in one condition and $M=0.61$ in the other condition.

\section{Habituation Effect and False Positive Explanation}

These two explanations predict that there will be no significant main or interaction effects involving the message condition.

\section{Robustness Check}

We will also conduct a robustness check in which we dichotomize belief in a just world using a median split to address concerns that our measure of belief in a just world is not perfectly normally distributed.

\section{Study 2}


Study 2 is a direct replication of Feinberg and Willer's (2011) second study. The goal of Study 2 is to test the role of belief in a just world with the original materials. Note that all participants watched a dire message so that only the effect of dire messages depending on participants' belief in a just world can be estimated.

\section{Method}

\section{Participants}

We will recruit 1802 participants from Amazon Mechanical Turk. We conducted a priori power analyses for the difference between two independent means using G*Power (Faul et al., 2009). We set the effect size in the power analysis to Cohen's $d=0.17$, a quarter of the effect size in the original study Cohen's $d=0.68$. A final sample size of 1802 participants will provide us with approximately $95 \%$ power given $\alpha=.05$ for a two-tailed test.

Instead of recruiting participants from craigslist, we will follow the same data collection strategy as in Study 1. That is, we will recruit participants from a large panel of previously recruited MTurk workers. Participants will be excluded participants if they have duplicate IDs (keeping only the first case), fail at least one out of our two attention checks completed pre-treatment-assignment, leave the study before they are randomly assigned to a condition, or have missing values for our measures of belief in a just world or belief in climate change. To ensure sufficient variance in belief in climate change, the sample will be recruited with soft quotas for participants' political ideology: 40\% liberal, $20 \%$ moderate, and $40 \%$ conservative.

\section{Materials and Procedure}

The materials and procedure are taken from Study 2 of Feinberg and Willer (2011). Any deviations from the original study are explicitly mentioned. First, participants will complete a questionnaire containing demographics and attention checks. Then they will be presented with an 
ostensible language comprehension test that provides participants with 14 scrambled six-word sentences and asks participants to form coherent sentences using five out of the six words per sentence. The goal of this task is to prime participants with the belief that the world is just or unjust. In the just world condition, participants received eight sentences describing the world as just, stable and predictable. In the unjust world condition, participants received eight sentences describing the world as unjust, unstable and unpredictable.

Afterwards, participants in all conditions will watch the same 60 -s video. The video has a dire message presenting climate change as an imminent catastrophe that will affect the children of the world the most. Finally, participants will complete the same seven-item measure of belief in climate change as in Study 1. Because our focus is on belief in climate change, we will not collect the measure of willingness to reduce carbon footprint that was collected in the original study. More details on the manipulation and measures are available in the Supplementary Materials.

\section{Results}

\section{Test of the Feinberg and Willer model.}

The Feinberg and Willer model predicts that participants in the just world condition will believe significantly less in climate change than participants in the unjust world condition.

\section{Ceiling Effect}

We will test if the level of belief in climate change was higher than in the original studies and if there was sufficient room for increases in belief in climate change. In Feinberg and Willer's (2011) first study, the average belief in climate change was $M=0.73$ before the treatment and $M=0.74$ after the treatment. In Feinberg and Willer's (2011) second study, the 
average belief in climate change was $M=0.74$ in one condition and $M=0.61$ in the other condition.

\section{Test of the Extended Parallel Process Model, Habituation Effect, and False Positive}

\section{Explanation}

These three explanations predict that there will be no significant main effect of the prime condition.

\section{Studies 3 - 5}

An important limitation of both studies in Feinberg and Willer (2011) is that they do not independently manipulate the direness and solvability of climate change messages. To address this concern and estimate the causal effects of both dire and solvable messages, we conducted three conceptual replication studies, Studies 3 to 5, that separately manipulated the direness and solvability of climate change messages. We report the pooled results across the three studies to maximize power. The study-specific results are consistent with the pooled results reported here (see Tables SM1 and SM2 in the Supplementary Materials).

\section{Method}

\section{Participants}

Across the three studies, we recruited 2307 participants from a large panel of previously recruited MTurk workers. We excluded participants if they had duplicate IDs $(n=45$; keeping only the first case), left the study before they were randomly assigned to a condition $(n=26)$, or had missing values for belief in a just world or belief in climate change $(n=66)$. The remaining 2170 participants consisted of 1211 men, 947 women, and 12 who did not answer the gender question. The average age was 40.7 years $(S D=13.2)$. The sample was $78 \%$ White, $8 \%$ African-American, 6\% Asian-American, 5\% Hispanic, 2\% other ethnicities, and 1\% did not 
answer the question. To ensure sufficient variance in belief in climate change, the sample was recruited to be balanced ideologically: $42 \%$ liberal, $21 \%$ moderate, and $37 \%$ conservative.

We conducted sensitivity power analyses $\mathrm{G}^{*}$ Power for fixed effects interactions (Faul et al., 2009). The final sample size of 2170 participants will provide us with approximately $95 \%$ power to detect interaction effects of $f \geq 0.08$ and approximately $80 \%$ to detect interaction effects of $f \geq 0.07$, given $\alpha=.05$ and a numerator degree of freedom of 1 .

\section{Materials and Procedure}

In all three studies, participants first completed the same measure of belief in a just world as in Study 1 (average Cronbach's $\alpha=.88$ ). Next, participants were randomly assigned to an experimental condition. Each experiment had a between-subjects manipulation of the severity of the consequences of climate. Study 3 had a medium problem condition (i.e., a message that described climate change as an important issue) and a dire problem condition (i.e., a message that described climate change as one of the biggest issues of our time). Study 4 had a control problem condition (i.e., a message that did not mention the severity of climate change) and a dire problem condition. Study 5 had a control problem condition, a dire problem condition, and an apocalyptic problem condition (i.e., a message that described climate change as the biggest natural threat to life as we know it).

Each experiment also had a between-subjects manipulation of the solvability of climate change. Study 3 had an impossible solution condition (i.e., a message that said climate change would continue to impact the world in the future) and a possible solution condition (i.e., a message that said climate change is still reversible). Studies 4 and 5 had a control solution condition (i.e., a message that did not mention the solvability of climate change) and a possible 
solution condition. Below, we analyze the data as coming from a 2 (problem condition: less dire vs. more dire $\left.{ }^{2}\right)$ x 2 (solution condition: less solvable vs. more solvable) between-subjects design.

Afterwards, participants completed a measure of belief in climate change (average Cronbach's $\alpha=.89$ ). In Study 3, we measured emotional reactions of fear and hope and the perceived solvability of climate change. In Studies 4 and 5, we measured the argued threat of climate change and the argued efficacy to address climate change. Finally, we collected demographic information and attention checks. More details on the manipulation and measures are available in the Supplementary Materials.

\section{Results}

Because the participants were recruited for different studies at different time points, we controlled for study in our regression models.

\section{Test of the Feinberg and Willer Model}

This model predicts a three-way belief in a just world $\mathrm{x}$ problem condition $\mathrm{x}$ solution condition interaction effect on belief in climate change. However, this interaction effect was essentially zero, non-significant, and explained less than $0.1 \%$ variance in belief in climate change, $b=-0.001, S E=0.02, t(2160)=-0.04, p=.965,95 \% \mathrm{CI}$ for $\mathrm{b}=[-0.05,0.05]$. Further, this model predicts the following pattern of simple effect. First, participants with a weak belief in a just world are not affected by the problem condition and the solution condition. Second, among participants with a strong belief in a just world in the less solvable solution condition, participants in the more dire problem condition will believe less in climate change than participants in the less dire problem condition. Third, among participants with a strong belief in a just world in the more solvable solution condition, participants in the more dire problem

\footnotetext{
${ }^{2}$ In Study 5, we categorized the dire problem condition and the apocalyptic problem condition into the more dire condition.
} 
condition will believe more in climate change than participants in the less dire problem condition. In contrast to these predictions, there was no evidence that dire messages affected belief in climate change among participants low or high in belief in a just world and in the less or more solvable solution condition (see Table 1). In short, the Feinberg and Willer model was not supported by Studies 3 to 5 .

\section{Test of the Extended Parallel Process Model}

This model predicts a problem condition x solution condition interaction effect on change in belief in climate change. However, this interaction effect was close to zero, non-significant, and explained less than $0.1 \%$ variance in belief in climate change, $b=0.01, S E=0.02, t(2163)=$ $0.38, p=.700,95 \% \mathrm{CI}$ for $\mathrm{b}=[-0.04,0.06]$. Further, this model predicts the following pattern of simple effect. First, among participants in the less solvable solution condition, participants in the more dire problem condition will believe less in climate change than participants in the less dire problem condition. Second, among participants in the more solvable solution condition, participants in the more dire problem condition will believe more in climate change than participants in the less dire problem condition. In contrast to these predictions, there was no evidence that dire messages affected belief in climate change among participants in the less or more solvable solution condition (see Table 2). In short, the EPPM was not supported by Studies 3 to 5 .

\section{Ceiling Effect}

The results are inconsistent with a ceiling effect. In Feinberg and Willer's (2011) first study, the average belief in climate change was $M=0.73$ before the treatment and $M=0.74$ after the treatment. In Feinberg and Willer's (2011) second study, the average belief in climate change was $M=0.74$ in one condition and $M=0.61$ in the other condition. In the current study, the 
average belief in climate change was $M=0.70$. Thus, inconsistent with a ceiling effect explanation, the average of belief in climate change was similar as in the original studies and there was sufficient room for increases in belief in climate change.

\section{Test of the Habituation Effect and False Positive Explanation}

These two explanations predict that the original effects would not be replicated. Results were consistent with this prediction.

\section{Robustness Check}

We conducted a robustness check in which we dichotomized belief in a just world using a median split to address concerns because our data suggested that our measure of belief in a just world was not perfectly normally distributed. In this robustness check, neither the belief in a just world $\mathrm{x}$ problem condition $\mathrm{x}$ problem condition $\mathrm{x}$ solution condition interaction effects, $F(1$, $2149)=0.25, p=.620$, nor the problem condition $\mathrm{x}$ solution condition interaction effect, $F(1$, $2152)=0.00, p=980$, were significant. Thus, the results of this robustness check were consistent with the results of our main analyses.

\section{Mechanisms}

The lack of support for many of the prevalent findings in this literature raises the question why dire and solvable messages did not affect belief in climate change. A critic might argue that our manipulations were simply ineffective operationalizations of dire and solvable messages. We think that this explanation is unlikely for several reasons. First, we used messages similar to the original messages used by Feinberg and Willer (2011). Second, the messages moved several cognitive and emotional measures as we show below. Note that the sample sizes for these analyses differ as these measures were not used in all three studies. 
The problem manipulation significantly increased the argued threat of climate change. Test statistics are provided in Table 3. Participants in the more dire problem condition reported that the message portrayed climate change as a significantly bigger problem than participants in the less dire problem condition. Participants in the more dire problem condition also reported that the message portrayed climate change as significantly less solvable than participants in the less dire problem condition, but this effect was much smaller than the effect on the argued threat of climate change. Notably, however, participants in the more dire problem condition did not report significantly more fear than participants in the less dire problem condition. The lack of significance might be due to a power issue because we only collected a measure of fear in Study 3 but not in Studies 4 and 5. Participants in the more dire problem condition also did not report significantly more hope than participants in the less dire problem condition. These results suggest that dire messages that increase the argued threat of climate change do not affect belief in climate change.

Our solution manipulations significantly increased the argued efficacy to address climate change and experiences of hope. Test statistics are provided in Table 3. Participants in the more solvable solution condition reported that the message portrayed climate change as significantly more solvable than participants in the less solvable solution condition. Participants in the more solvable solution condition also reported feeling significantly more hope and less fear than participants in the less solvable solution condition. Participants in the more solvable solution condition reported that the message portrayed climate change as a significantly bigger problem than participants in the less solvable solution condition, but this effect was much smaller than the other effects. These results suggest that solvable messages that increase the argued efficacy to address climate change and hope and decrease fear do not affect belief in climate change. 
Overall, these results suggest that the failed effects of dire and solvable messages cannot be attributed to failed operationalizations of dire and solvable messages.

\section{Study 6}

Study 6 tests the effects of dire and solvable messages on a new, relatively unknown environmental problem with potential dire consequences. This study allows a test of the habituation effect.

\section{Method}

\section{Participants}

We will recruit 2000 participants from Amazon Mechanical Turk. We conducted a priori power analyses for fixed effects interactions using G*Power (Faul et al., 2009). A final sample size of 2000 participants will provide us with approximately $95 \%$ power to detect interaction effects of $f \geq 0.09$ and approximately $80 \%$ to detect interaction effects of $f \geq 0.07$, given $\alpha=.05$ and a numerator degree of freedom of 1.

As in the prior studies, we will recruit participants from a large panel of previously recruited MTurk workers. Participants will be excluded participants if they have duplicate IDs (keeping only the first case), fail at least one out of our two attention checks completed pre-treatment-assignment, leave the study before they are randomly assigned to a condition, or have missing values for our measures of belief in a just world or skepticism regarding climate change. To ensure sufficient variance in belief in climate change, the sample will be recruited with soft quotas for participants' political ideology: 40\% liberal, 20\% moderate, and 40\% conservative.

\section{Materials and Procedure}


Participants will complete a questionnaire containing demographics, attention checks, and the same measure of belief in a just world as in Study 1 before being randomly assigned to one of four conditions. The experiment will have a 2 (problem condition: medium vs. dire) x 2 (solution condition: impossible vs. possible) between-subjects design. Every participant will read an essay about the effects of toxic chemicals on human reproduction (based on Brokovich, 2021). Participants in the dire problem condition will read information suggesting that toxic chemicals in our environment threaten humanity by causing a reproductive crisis. Participants in the medium problem condition will read similar information with less dire language. Participants in the possible solution condition will read additional information suggesting that the problem of toxic chemicals can be addressed. Participants in the impossible solution condition will read additional information suggesting that the problem of toxic chemicals cannot be sufficiently addressed. After the manipulation, participants will complete a seven-item measure of belief in toxic chemicals. In addition, we will collect exploratory three-item measures of the argued threat of toxic chemicals and argued efficacy to address toxic chemicals as well as participants' experiences of fear and hope. More details on the manipulation and measures are available in the Supplementary Materials.

\section{Results}

\section{Test of the Feinberg and Willer Model}

This model predicts a three-way belief in a just world $\mathrm{x}$ problem condition $\mathrm{x}$ solution condition interaction effect on belief in toxic chemicals. Further, this model predicts the following pattern of simple effects. First, participants with a weak belief in a just world are not affected by the problem condition and the solution condition. Second, among participants with a strong belief in a just world in the impossible solution condition, participants in the dire problem 
condition will believe less in the toxic chemicals than participants in the medium problem condition. Third, among participants with a strong belief in a just world in the possible solution condition, participants in the dire problem condition will believe more in toxic chemicals than participants in the medium problem condition.

\section{Test of the Extended Parallel Process Model}

This model predicts a problem condition $\mathrm{x}$ solution condition interaction effect on belief in toxic chemicals. Further, this model predicts the following pattern of simple effects. First, among participants in the impossible solution condition, participants in the dire problem condition will believe less in toxic chemicals than participants in the medium problem condition. Second, among participants in the possible solution condition, participants in the dire problem condition will believe more in toxic chemicals than participants in the medium problem condition.

\section{Test of the Habituation Effect}

The habituation hypothesis predicts that, on this new issue, the effects of dire and solvable messages will be non-zero.

\section{Test of the False Positive Explanation}

The false positive hypothesis predicts that all effects of dire and solvable messages will be zero.

\section{Robustness Check}

We will also conduct a robustness check in which we dichotomize belief in a just world using a median split to address concerns that belief in a just world is not perfectly normally distributed. 
Figure 1: The Feinberg \& Willer (2011) Model

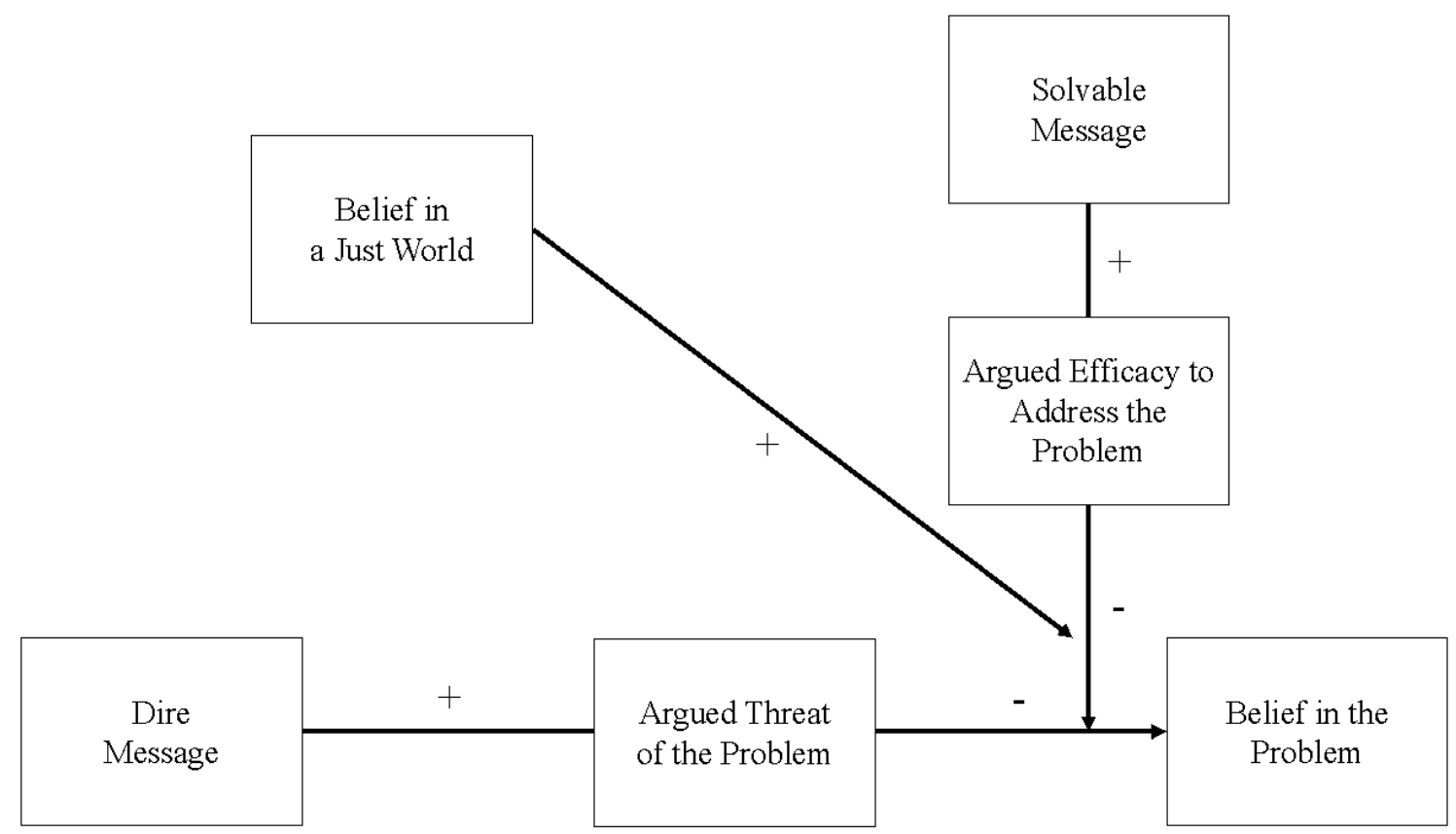


Figure 2 : The Extended Parallel Process Model

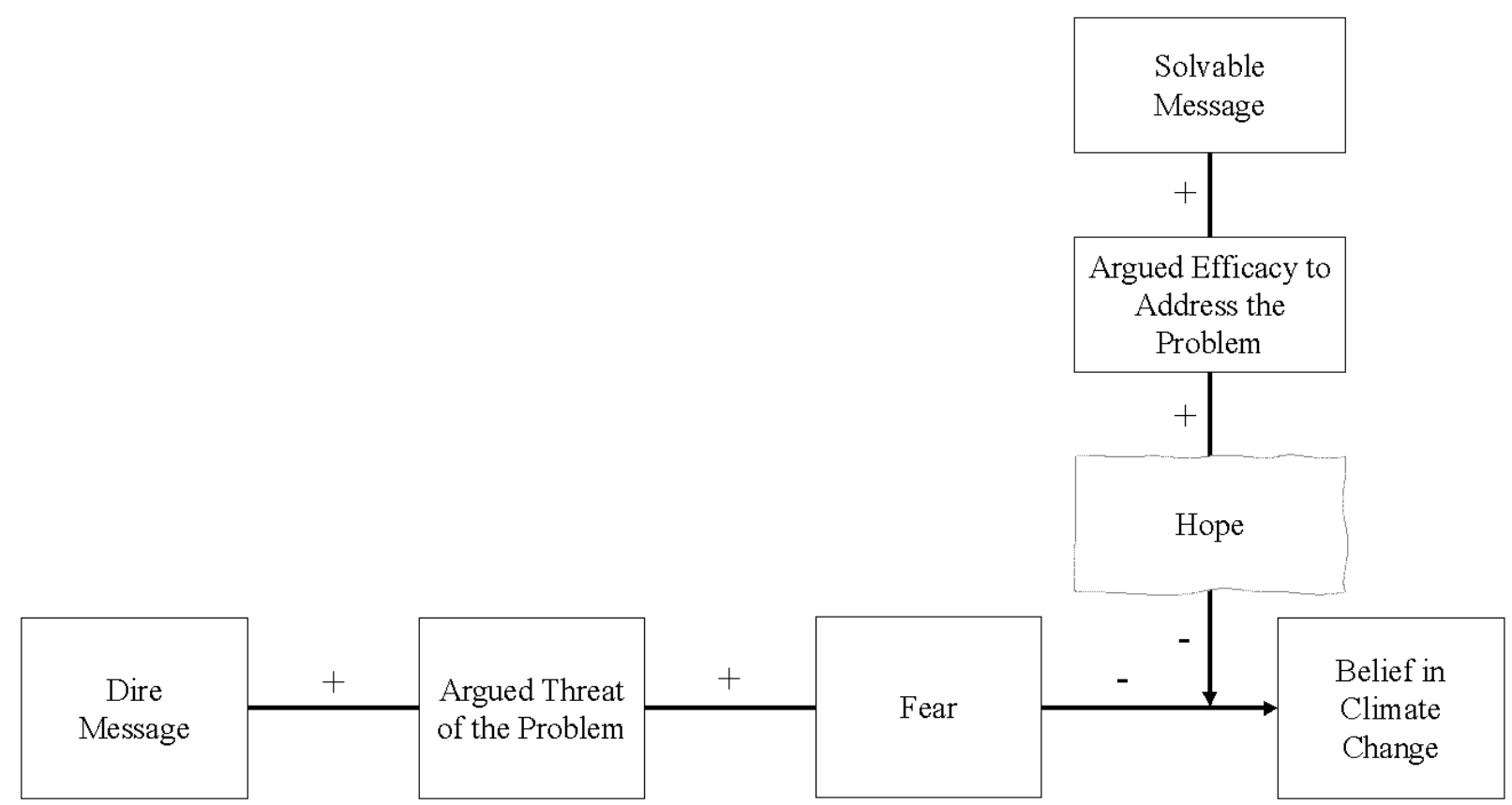

Note. The link between argued efficacy and hope is depicted differently because it is not explicitly mentioned in the original model. 
Table 1: Test of the Feinberg and Willer Model

Test Statistics

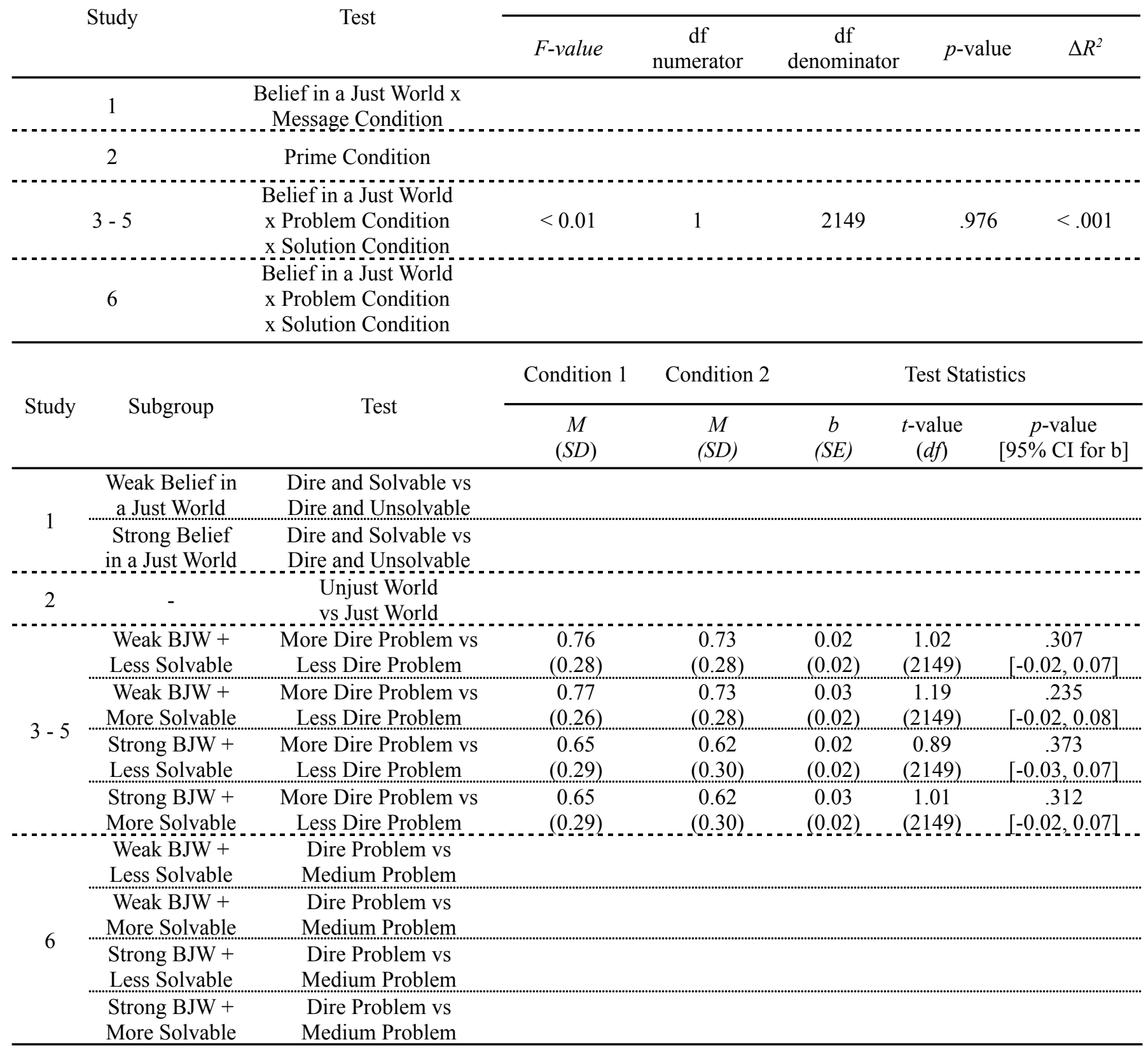

Notes. Regression coefficients and t-tests based on linear regression analyses. In all studies, we controlled for gender, age, race, and education. For the Studies 3-5 analyses, we also controlled for study. For means and standard deviations in subgroups based on belief in a just world, we dichotomized belief in a just world via a median split. 
Table 2: Test of the Extended Parallel Process Model

Test Statistics

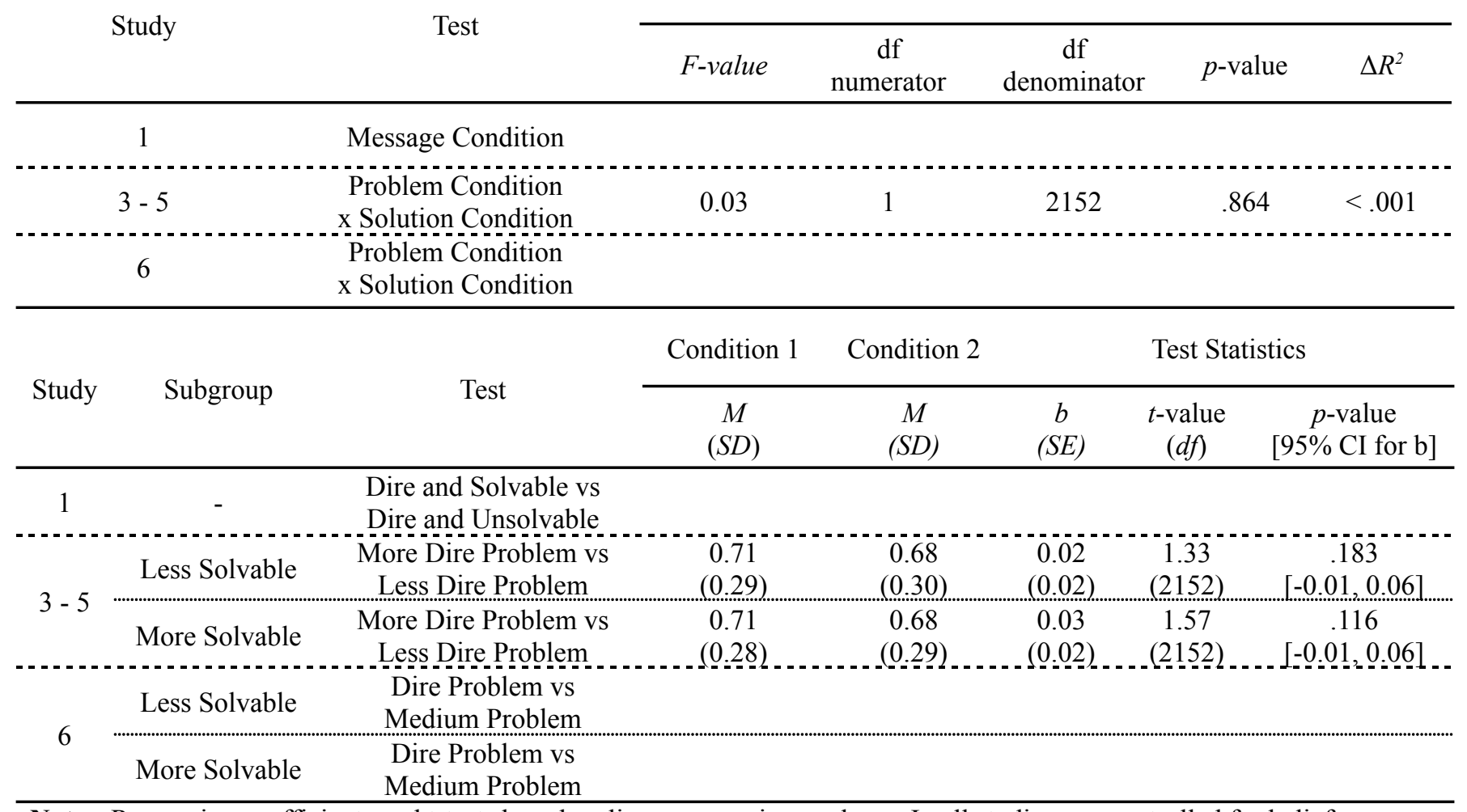

Notes. Regression coefficients and t-tests based on linear regression analyses. In all studies, we controlled for belief in a just world, gender, age, race, and education. For the Studies 3-5 analyses, we also controlled for study. 
Table 3: Effects of Dire and Solvable Messages on Mechanisms

\begin{tabular}{|c|c|c|c|c|c|c|c|}
\hline \multirow{2}{*}{ DV } & \multirow{2}{*}{ Study } & \multirow{2}{*}{ Test } & \multirow{2}{*}{$\begin{array}{c}\text { Condition } 1 \\
M \\
(S D) \\
\end{array}$} & \multirow{2}{*}{$\begin{array}{c}\text { Condition } 2 \\
M \\
(S D) \\
\end{array}$} & \multicolumn{3}{|c|}{ Test Statistics } \\
\hline & & & & & $\begin{array}{c}b \\
(S E) \\
\end{array}$ & $\begin{array}{l}t \text {-value } \\
(d f)\end{array}$ & $\begin{array}{c}p \text {-value } \\
{[95 \% \mathrm{CI} \text { for } \mathrm{b}]}\end{array}$ \\
\hline \multirow{5}{*}{$\begin{array}{l}\text { Argued } \\
\text { Threat of } \\
\text { Climate } \\
\text { Change }\end{array}$} & 1 & $\begin{array}{l}\text { Dire and Solvable vs } \\
\text { Dire and Unsolvable }\end{array}$ & & & & & \\
\hline & $4-5$ & $\begin{array}{l}\text { More Dire Problem vs } \\
\text { Less Dire Problem }\end{array}$ & $\begin{array}{c}0.90 \\
(0.18)\end{array}$ & $\begin{array}{c}0.62 \\
(0.30)\end{array}$ & $\begin{array}{c}0.28 \\
(0.01)\end{array}$ & $\begin{array}{c}22.94 \\
(1625)\end{array}$ & $\begin{array}{c}<.001 \\
{[0.26,0.30]}\end{array}$ \\
\hline & $4-5$ & $\begin{array}{l}\text { More Solvable Solution vs } \\
\text { Less Solvable Solution }\end{array}$ & $\begin{array}{c}0.81 \\
(0.23)\end{array}$ & $\begin{array}{c}0.75 \\
(0.32)\end{array}$ & $\begin{array}{c}0.06 \\
(0.01)\end{array}$ & $\begin{array}{c}5.30 \\
(1625)\end{array}$ & $\begin{array}{c}<.001 \\
{[0.04,0.091}\end{array}$ \\
\hline & 6 & $\begin{array}{l}\text { Dire Problem vs } \\
\text { Medium Problem }\end{array}$ & & & & & \\
\hline & 6 & $\begin{array}{l}\text { Possible Solution vs } \\
\text { Impossible Solution }\end{array}$ & & & & & \\
\hline \multirow{5}{*}{$\begin{array}{c}\text { Argued } \\
\text { Efficacy to } \\
\text { Address } \\
\text { Climate } \\
\text { Change }\end{array}$} & 1 & $\begin{array}{l}\text { Dire and Solvable } \\
\text { vs Dire and Unsolvable }\end{array}$ & & & & & \\
\hline & $4-5$ & $\begin{array}{l}\text { More Dire Problem vs } \\
\text { Less Dire Problem }\end{array}$ & $\begin{array}{c}0.58 \\
(0.33)\end{array}$ & $\begin{array}{c}0.58 \\
(0.37)\end{array}$ & $\begin{array}{l}-0.03 \\
(0.01)\end{array}$ & $\begin{array}{l}-2.41 \\
(1627)\end{array}$ & $\begin{array}{c}.016 \\
{[-0.06,-0.01] \ldots}\end{array}$ \\
\hline & $4-5$ & $\begin{array}{l}\text { More Solvable Solution vs } \\
\text { Less Solvable Solution }\end{array}$ & $\begin{array}{c}0.81 \\
(0.22)\end{array}$ & $\begin{array}{c}0.35 \\
(0.30)\end{array}$ & $\begin{array}{c}0.46 \\
(0.01)\end{array}$ & $\begin{array}{l}36.91 \\
(1627)\end{array}$ & $\begin{array}{c}<.001 \\
{[0.44,0.491}\end{array}$ \\
\hline & 6 & $\begin{array}{l}\text { Dire Problem vs } \\
\text { Medium Problem }\end{array}$ & & & & & \\
\hline & 6 & $\begin{array}{l}\text { Possible Solution vs } \\
\text { Impossible Solution }\end{array}$ & & & & & \\
\hline \multirow{5}{*}{ Fear } & 1 & $\begin{array}{l}\text { Dire and Solvable vs } \\
\text { Dire and Unsolvable }\end{array}$ & & & & & \\
\hline & 3 & $\begin{array}{l}\text { More Dire Problem vs } \\
\text { Less Dire Problem }\end{array}$ & $\begin{array}{c}0.48 \\
(0.30)\end{array}$ & $\begin{array}{c}0 . .45 \\
(0.30)\end{array}$ & $\begin{array}{c}0.04 \\
(0.03)\end{array}$ & $\begin{array}{r}1.60 \\
(508)\end{array}$ & $\begin{array}{c}.111 \\
{[-0.01,0.091]}\end{array}$ \\
\hline & 3 & $\begin{array}{l}\text { More Solvable Solution vs } \\
\text { Less Solvable Solution }\end{array}$ & $\begin{array}{c}0.22 \\
(0.23)\end{array}$ & $\begin{array}{c}0.44 \\
(0.29)\end{array}$ & $\begin{array}{l}-0.22 \\
(0.02)\end{array}$ & $\begin{array}{l}-9.63 \\
(508)\end{array}$ & $\begin{array}{c}<.001 \\
{[-0.08,0.01]}\end{array}$ \\
\hline & 6 & $\begin{array}{l}\text { Dire Problem vs } \\
\text { Medium Problem }\end{array}$ & & & & & \\
\hline & 6 & $\begin{array}{l}\text { Possible Solution vs } \\
\text { Impossible Solution }\end{array}$ & & & & & \\
\hline \multirow{5}{*}{ Hope } & 1 & $\begin{array}{l}\text { Dire and Solvable vs } \\
\text { Dire and Unsolvable }\end{array}$ & & & & & \\
\hline & 3 & $\begin{array}{l}\text { More Dire Problem vs } \\
\text { Less Dire Problem }\end{array}$ & $\begin{array}{c}0.22 \\
(0.24)\end{array}$ & $\begin{array}{c}0.25 \\
(0.26)\end{array}$ & $\begin{array}{l}-0.04 \\
(0.02)\end{array}$ & $\begin{array}{l}-1.75 \\
(508)\end{array}$ & $\begin{array}{c}.081 \\
{[-0.08,0.00]}\end{array}$ \\
\hline & 3 & $\begin{array}{l}\text { More Solvable Solution vs } \\
\text { Less Solvable Solution }\end{array}$ & $\begin{array}{c}0.58 \\
(0.28)\end{array}$ & $\begin{array}{c}0.29 \\
(0.26)\end{array}$ & $\begin{array}{c}0.30 \\
(0.02)\end{array}$ & $\begin{array}{l}12.98 \\
(508)\end{array}$ & $\begin{array}{c}<.001 \\
{[0.26,0.35]}\end{array}$ \\
\hline & 6 & $\begin{array}{l}\text { Dire Problem vs } \\
\text { Medium Problem }\end{array}$ & & & & & \\
\hline & 6 & $\begin{array}{l}\text { Possible Solution vs } \\
\text { Impossible Solution }\end{array}$ & & & & & \\
\hline
\end{tabular}

Notes. Regression coefficients and t-tests based on linear regression analyses. In all studies, we controlled for belief in a just world, gender, age, race, and education. For the Studies 4-5 analyses, we also controlled for study. 


\section{References}

Al Jazeera (2020). Declare 'state of climate emergency', UN boss urges world leaders. Retrieved from https://www.aljazeera.com/news/2020/12/12/countries-must-declare-climateemergency-un-chief

Ballew, M. T., Leiserowitz, A., Roser-Renouf, C., Rosenthal, S. A., Kotcher, J. E., Marlon, J. R., Lyon, E., Goldberg, M. H., \& Maibach, E. W. (2019). Climate change in the American mind: Data, tools, and trends. Environment: Science and Policy for Sustainable Development, 61(3), 4-18. doi:10.1080/00139157.2019.1589300

Brockovich, E. (2021). Plummeting sperm counts, shrinking penises: toxic chemicals threaten humanity. Retrieved from https://www.theguardian.com/commentisfree/2021/mar/18/toxicchemicals-health-humanity-erin-brokovich

Clifford, S., Jewell, R. M., \& Waggoner, P. D. (2015). Are samples drawn from Mechanical Turk valid for research on political ideology?. Research \& Politics, 2(4), 2053168015622072. doi:10.1177/2053168015622072

Coppock, A. (2019). Generalizing from survey experiments conducted on Mechanical Turk: A replication approach. Political Science Research and Methods, 7(3), 613-628. doi:10.1017/psrm.2018.10

Dalbert, C., Montada, L., \& Schmitt, M. (1987). Glaube an eine gerechte welt als motiv: Validierungskorrelate zweier Skalen [Belief in a just world as motive: Validity correlates of two scales]. Psychologische Beitrage, 29, 596-615.

Faul, F., Erdfelder, E., Buchner, A., \& Lang, A. G. (2009). Statistical power analyses using G* Power 3.1: Tests for correlation and regression analyses. Behavior Research Methods, 41(4), 1149-1160. doi:10.3758/BRM.41.4.1149 
Feinberg, M., \& Willer, R. (2011). Apocalypse Soon?: Dire Messages Reduce Belief in Global Warming by Contradicting Just-World Beliefs. Psychological Science, 22(1), 34-38. doi:10.1177/0956797610391911

Feldman, L., \& Hart, P. S. (2016). Using political efficacy messages to increase climate activism: The mediating role of emotions. Science Communication, 38(1), 99-127. doi:10.1177/1075547015617941

Groves, P. M., \& Thompson, R. F. (1970). Habituation: a dual-process theory. Psychological Review, 77(5), 419-450. doi:10.1037/h0029810

Hauser, D. J., \& Schwarz, N. (2016). Attentive Turkers: MTurk participants perform better on online attention checks than do subject pool participants. Behavior Research Methods, 48(1), 400-407. doi:10.3758/s13428-015-0578-z

Hornsey, M. J., \& Fielding, K. S. (2017). Attitude roots and Jiu Jitsu persuasion: Understanding and overcoming the motivated rejection of science. American Psychologist, 72(5), 459-473. doi:10.1037/a0040437

Intergovernmental Panel on Climate Change (2014). Climate change 2014: Synthesis report.

Retrieved from https://www.ipcc.ch/report/ar5/syr/

Klein, R. A., Vianello, M., Hasselman, F., Adams, B. G., Adams Jr, R. B., Alper, S., ... \& Batra, R. (2018). Many Labs 2: Investigating variation in replicability across samples and settings. Advances in Methods and Practices in Psychological Science, 1(4), 443-490. doi: $10.1177 / 2515245918810225$

Markowitz, E. M., \& Shariff, A. F. (2012). Climate change and moral judgement. Nature Climate Change, 2(4), 243-247. doi:10.1038/nclimate1378

McCright, A. M., Charters, M., Dentzman, K., \& Dietz, T. (2016). Examining the effectiveness 
of climate change frames in the face of a climate change denial counter-frame. Topics in Cognitive Science, 8(1), 76-97. doi:10.1111/tops.12171

O'Neill, S., \& Nicholson-Cole, S. (2009). "Fear won't do it" promoting positive engagement with climate change through visual and iconic representations. Science Communication, 30(3), 355-379. doi:10.1177/1075547008329201

Open Science Collaboration. (2015). Estimating the reproducibility of psychological science. Science, 349(6251). doi:10.1126/science.aac4716

Pearce, W., Brown, B., Nerlich, B., \& Koteyko, N. (2015). Communicating climate change: conduits, content, and consensus. Wiley Interdisciplinary Reviews: Climate change, 6(6), 613-626. doi:10.1002/wcc.366

Peters, G. J. Y., Ruiter, R. A., \& Kok, G. (2013). Threatening communication: a critical re-analysis and a revised meta-analytic test of fear appeal theory. Health Psychology Review, 7(sup1), S8-S31. doi:10.1080/17437199.2012.703527

Petty, R. E., \& Cacioppo, J. T. (1984). The effects of involvement on responses to argument quantity and quality: Central and peripheral routes to persuasion. Journal of Personality and Social Psychology, 46(1), 69-81. doi:10.1037/0022-3514.46.1.69

Stern, P. C. (2012). Fear and hope in climate messages. Nature Climate Change, 2(8), 572-573. doi:10.1038/nclimate1610

The New York Times Editorial Board (2019, December 7). The world solved the ozone problem. It can solve climate change. The New York Times. Retrieved from https://www.nytimes.com/2019/12/07/opinion/sunday/ozone-climate-change.html

Van Boven, L., Ehret, P. J., \& Sherman, D. K. (2018). Psychological barriers to bipartisan public support for climate policy. Perspectives on Psychological Science, 13(4), 492-507. 
doi:10.1177/1745691617748966

Wallace-Wells, D. (2017). The uninhabitable Earth, annotated edition. Intelligencer. Retrieved from https://nymag.com/intelligencer/2017/07/climate-change-earth-too-hot-forhumans-annotated.html

Westoby, R., \& McNamara, K. E. (2019). Fear, grief, hope and action. Nature Climate Change, 9(7), 500-501. doi:10.1038/s41558-019-0511-z

Witte, K. (1992). Putting the fear back into fear appeals: The extended parallel process model. Communication Monographs, 59(4), 329-349. doi:10.1080/03637759209376276

Witte, K., \& Allen, M. (2000). A meta-analysis of fear appeals: Implications for effective public health campaigns. Health Education \& Behavior, 27(5), 591-615. doi:10.1177/109019810002700506

Wong-Parodi, G., \& Feygina, I. (2020). Understanding and countering the motivated roots of climate change denial. Current Opinion in Environmental Sustainability, 42, 60-64. doi:10.1016/j.cosust.2019.11.008 